\title{
The combined automatic repeat request and rate control mechanism in S-band mobile interactive multimedia asynchronous return link- design background, parameter dimensioning and first experimental results
}

\author{
Cristina Párraga Niebla* ${ }^{* \dagger}$ and Alessandro Del Bianco \\ DLR Institute of Communications and Navigation, Oberpfaffenhofen, Germany
}

\begin{abstract}
SUMMARY
In the S-band mobile interactive multimedia specification, an asynchronous access using Enhanced Spread Spectrum Aloha random access has been defined for occasional messaging applications. This access scheme is complemented by a combined automatic repeat request and terminal rate control mechanism that is described in this paper. Furthermore, a queuing theory-based model is developed to describe the behaviour of the automatic repeat request mechanism, and performance results from the first proof-of-concept implementation are presented. Copyright () 2014 John Wiley \& Sons, Ltd.
\end{abstract}

Received 20 February 2013; Revised 20 February 2014; Accepted 21 February 2014

KEY WORDS: mobile satellite service; S-band; reliably messaging; ARQ; rate control; congestion control; queuing theory

\section{INTRODUCTION}

The S-band mobile interactive multimedia (S-MIM) specification published by the European Telecommunications Standards Institute (ETSI) [1] describes an integrated satellite/terrestrial mobile system to be operated in frequencies below $3 \mathrm{GHz}$, capable of providing a variety of services, such as interactive broadcast, messaging and bi-directional (near-) real-time services, including voice [2, 3]. In the forward link, S-MIM relies on any broadcast air interface that fulfils the requirements described in part 2 of [1], such as digital video broadcasting — satellite services to handheld device [4], preferably the enhanced version containing a low-latency profile [5]. In the return link, two different, non-exclusive air interfaces have been defined, each of them addressing a different set of services:

- Asynchronous access using Enhanced Spread Spectrum Aloha (ESSA) random access [6, 7], described in part 3 of [1], for both satellite and terrestrial components of the access network.

- Synchronous access using quasi-synchronous code division multiple access [8], described in part 4 of [1], for the satellite component only.

On the one hand, the synchronous access is better suited for bi-directional (near-) real-time applications. On the other hand, the asynchronous access is well suited for low rate low duty cycle messaging services, for example, to support interactive broadcast and machine-to-machine applications, such as industrial supervisory control and data acquisition systems, fleet management or containers tracking, automatic highway tollgates, traffic light controllers, or energy systems monitoring.

Even if messaging services may have relaxed delay requirements compared with real-time applications, the requirements in terms of error-free delivery are more stringent. The packet loss ratio performance of

*Correspondence to: Cristina Párraga Niebla, DLR Institute of Communications and Navigation, Oberpfaffenhofen, Germany.

${ }^{\dagger}$ E-mail: Cristina.Parraga@dlr.de 
ESSA is dependent on the load and received power distribution [6,7], as it is based on interference cancellation. For those applications that require higher transmission reliability than that provided by ESSA, an error control mechanism in the link layer has been defined in part 5 of [1], combined with provisions to keep the system load within manageable limits by the ESSA demodulator, that is, signalling mechanisms to configure the maximum terminal transmission rate from the satellite hub.

This paper focuses on the applied ARQ mechanism in S-MIM combined with terminal transmission rate control and supportive signalling to adapt the terminal transmission rate to the sensed load at the satellite hub. The remaining of the paper is organised as follows: Section 2 describes the combined ARQ and terminal rate control mechanism specified in part 5 of [1] and the related signalling framework. Section 3 develops a queuing theory model to describe the behaviour of the ARQ and rate control mechanism and provides parameter dimensioning examples using this model. Section 4 describes the issues found in the first real proof-of-concept implementation of the S-MIM specification with regard to the ARQ and rate control. Finally, Section 5 draws the conclusions.

\section{COMBINED AUTOMATIC REPEAT REQUEST AND RATE CONTROL MECHANISM FOR ENHANCED SPREAD SPECTRUM ALOHA}

\subsection{The basic principle}

With the aim at providing highly reliable messaging services, an ARQ mechanism combined with a terminal transmission rate control has been defined in S-MIM [1]. The first mechanism provides retransmissions in case of packet losses in the ESSA air interface, while the second allows the hub configuring the maximum rate at which terminals are allowed to transmit in order keep the traffic load levels under manageable limits by the ESSA demodulator. The integration of these two mechanisms is achieved by letting the satellite hub configures the parameters that control the ARQ mechanism at the terminals through signalling, that is, the minimum time between consecutive transmissions, the persistence index to acquire a transmission opportunity and the number of allowed retransmissions.

Figure 1 shows the basic principle of the integrated ARQ and transmission rate control mechanism at the link layer: the terminals send link layer packets to the satellite hub (depicted as LL packets in the figure) and those are acknowledged by the satellite hub if they are correctly received; in parallel, the satellite hub estimates continuously the traffic load in the channel and sets the signalling tables tailoring the ARQ parameters to the estimated load level to prevent or counteract congestion; the terminals receive the signalling tables and configure the ARQ parameters accordingly. While the ARQ mechanism and related signalling are specified in the S-MIM specification, the load control algorithms can be proprietary.

\subsection{Transmission modes and signalling}

S-band mobile interactive multimedia allows three different terminal transmission modes as listed in the succeeding text, to allow different guarantees in terms of reliability, so that each application can use the most suitable transmission mode with regard to its quality of service requirements:



Figure 1. Principle of combined automatic repeat request and transmission rate control. 
- Tr-mode: transparent transmission, that is, no link layer acknowledgement will be issued.

- ACK mode: acknowledged transmission, $\mathrm{CRC}^{\ddagger}$ (cyclic redundancy code) is used as hash for each link layer packet upon correct reception.

- uACK mode: unequivocally acknowledged transmission, that is, link layer acknowledgements in the form of a dedicated unicast ACK message will be issued by the satellite hub in the forward link upon correct reception.

The difference between the ACK and uACK modes lies on the probability of false detection (i.e. interpreting as own and ACK that actually refers to a different terminal or packet) versus the capacity required in the forward link.

In terms of signalling, the terminal specifies the transmission mode for each link layer packet in the link layer header, that is, the return link encapsulation header, more concretely in the 'alpdu_label', see part 5 of [1]. In the forward link, the S-MIM specification provides the 'SSA Access Table' that indicates the ARQ parameters for each load level and service class as listed in the succeeding text:

- 'Back_off_time' $\left(T_{b o t}\right)$ : minimum number of time units between consecutive link layer transmissions. A time unit corresponds to $10 \mathrm{~ms}$.

- 'Persistence_index' $(P)$ : the value from which the probability of transmitting at the next time step $P_{t x}(P)$ (in $10 \mathrm{~ms}$ steps), computed with the equation in the succeeding text.

$$
P_{t x}(P)=\frac{1}{2^{(P / 2)}}
$$

- 'Ack_time_out' $\left(T_{A C K}\right)$ : maximum waiting time for the reception of an acknowledgement.

- 'Max_retransmissions' $\left(N_{R e T x}\right)$ : maximum number of allowed retransmissions of the same packet.

It shall be noted that the currently estimated load level is specified in the 'SSA Signalling Load' table that can be found in part 5 of [1].

\subsection{A cumulative stop-and-wait automatic repeat request approach}

The selection of a suitable ARQ type for the S-MIM system has been significantly influenced by two established requirements: (i) the complexity at the terminal must be kept as low as possible and (ii) the capacity required at the forward link shall be kept an order of magnitude lower than the return link traffic.

It is well known that stop-and-wait (SW) ARQ is inefficient because of the idle times, while goback-N or selective repeat achieves higher throughput and the same reliability than SW; whereas SW is the simplest one [9]. Those well-known statements are in general true but not fully applicable to the messaging service scenario in S-MIM. Firstly, the idle periods of SW are only inefficient if the transmission buffer is filled at higher rate than the serving rate of SW. In case of occasional low rate messaging services, this is not applicable. Furthermore, idle periods will allow smoothing the interference caused by simultaneous transmissions by different terminals, turning this 'traditional' drawback into an advantage. Secondly, selective repeat and go-back-N require the existence of a sequence number at the link layer and sorted packet delivery. Both requirements are not fulfilled in S-MIM: on the one hand, the interference cancellation (IC) process may deliver packets in an unsorted manner depending on the level of interference to be cancelled to decode each packet; on the other hand, the return link encapsulation only permits differentiating whether a link layer packet is the first, last or any intermediate fragment of an Internet protocol (IP) packet but does not allow differentiating the order across different IP packets and does not allow differentiating the order of different intermediate fragments if the IP packet was fragmented into more than three-link layer fragments. Hybrid ARQ schemes [10] are discarded because the ESSA burst already includes forward error correction (FEC),

\footnotetext{
${ }^{\ddagger}$ Calculated as specified in part 5 of [1]. 
as turbo codes are applied to the data bursts, and additional FEC at the link layer is undesired because of the limited size of the data bursts.

Given the constraints imposed by the low complexity and low overhead requirements together with the peculiarities discussed earlier in the S-MIM system, a cross-layer variation of the SW ARQ mechanism has been defined for the asynchronous return link in the S-MIM system. It consist on a SW ARQ mechanism that acknowledges the correct reception of all link layer fragments of an IP packet in a cumulative acknowledgement and requires a retransmission of all fragments if the IP packet cannot be reconstructed out of the received fragments, that is, one or more are missing. This mechanism limits the forward link overhead (even more if the CRC-based acknowledgements are used) and shifts the complexity to the satellite hub, while the terminal only requires a limited retransmission buffer. This ARQ mechanism, to which we will refer with the term Cumulative SW $\mathrm{ARQ}$, is depicted in the form of flowchart in Figure 2.

As it can be observed, the scheduler schedules all fragments of an IP packet. The packets remain first on hold until they get a transmission opportunity. This process uses the persistence index $\mathrm{P}$ with the value indicated in the received 'SSA access table' to calculate the probability to get a transmission opportunity according to Equation (1). It shall be noted that the persistency check is done at IP level,

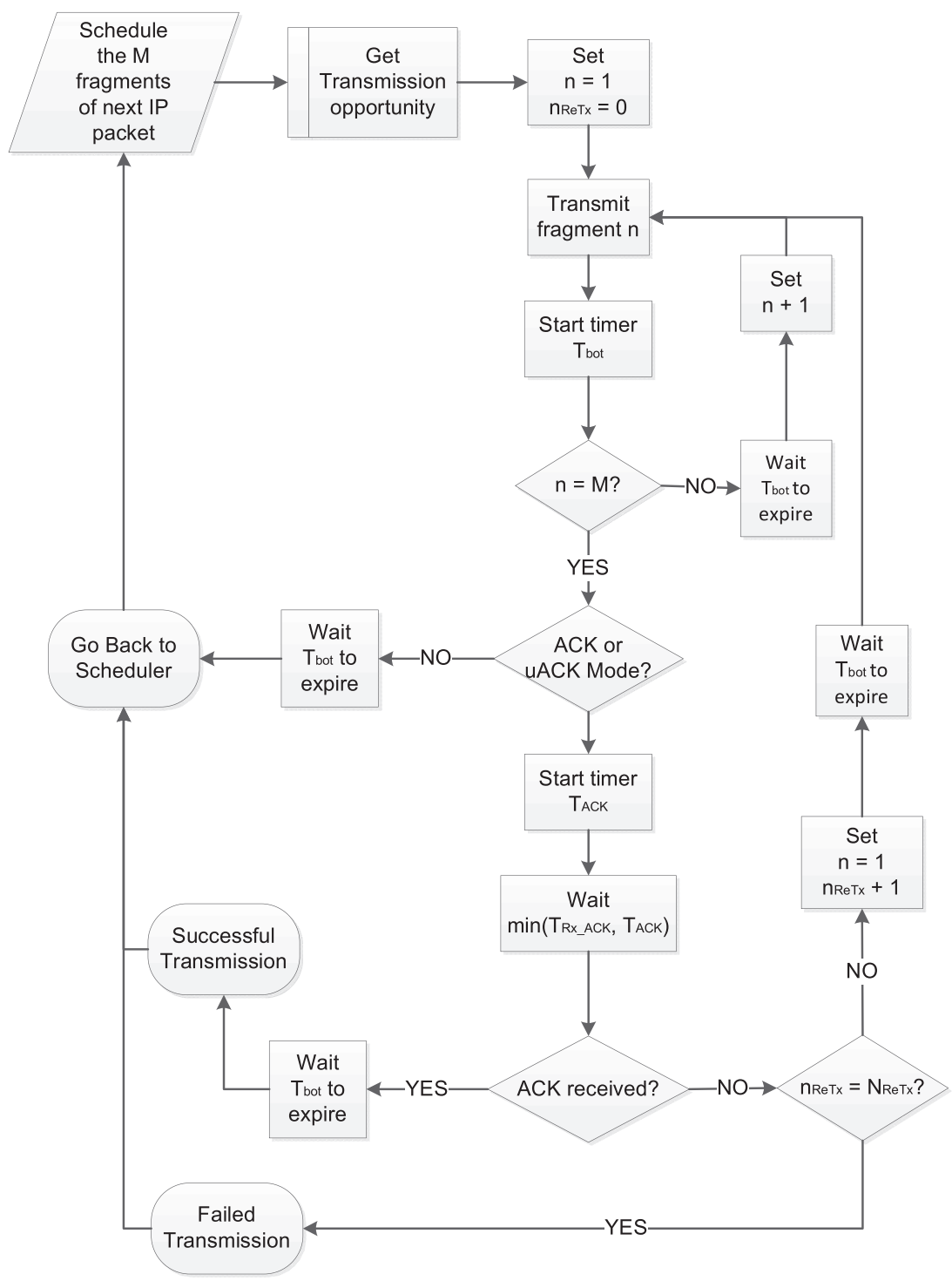

Figure 2. Automatic repeat request mechanism flowchart at the Enhanced Spread Spectrum Aloha receiver. 
not at fragment level. Basically, the process 'Get Transmission Opportunity' flips a coin to decide if the packets are passed or not to the ARQ mechanism. If it fails, the process waits a time equal to the $T_{b o t}$ specified in the 'SSA access table' and tries again. The process is repeated until a transmission opportunity is granted. When this happens, the link layer fragments of the current IP packet can be passed to the ARQ process.

Once the link layer fragments are passed to the ARQ process, the fragments are sent consecutively with a time gap of $T_{b o t}$ between consecutive fragments. If the related application selected the Tr-mode, no ACK is required, and the process goes back to the scheduler. On the contrary, if the ACK or uACK modes were selected, the timer to wait for the ACK is set to the value $T_{A C K}$ (indicated in the 'SSA access table') when the last fragment is transmitted. At the same time, a parallel timer is set to $T_{b o t}$. The system waits until the ACK is actually received, $T_{R x_{-} A C K}$ in Figure 2, or $T_{A C K}$ expires, whatever happens first. If the ACK was received before $T_{A C K}$ expired, the transmission is considered successful, and the process goes back to the scheduler as soon as $T_{b o t}$ expires. It shall be noted that, if $T_{A C K}>T_{b o t}, T_{b o t}$ may have expired already when the ACK is received; in this case, the waiting time for $T_{b o t}$ to expire is equal to 0 , and in practice, the process goes back directly to the scheduler. Otherwise, the process checks if retransmissions are still allowed, that is, if the number of retransmissions already sent for the current IP packet $\left(n_{R e T x}\right)$ is still below the maximum allowed as indicated by the parameter $N_{R e T x}$ in the 'SSA access table'. If retransmissions are still allowed, the process waits until $T_{b o t}$ expires (if it did not do it yet) to start with the retransmission. This process is repeated until the ACK is received or $N_{R e T x}$ is reached, in which case, the transmission is considered unsuccessful, and the process goes back to the scheduler.

\section{BEHAVIOUR MODELLING AND PARAMETER DIMENSIONING}

For the dimensioning of the ARQ parameters $T_{b o t}, P, N_{R e T x}$, and $T_{A C K}$ to meet quality of service requirements, it is worth describing the behaviour of the terminal transmission with the ARQ mechanism presented in the previous section. We define a metric to describe the time to successfully delivery an IP packet to the recipient: the successful delivery delay $T_{S D D}$.

\subsection{A queuing theory-based model}

We define the following parameters:

- $\lambda$ : packet rate per terminal, that is, the number of packets per second that is delivered from the upper layers to transmit towards the hub;

- $E(B)$ : expected serving time in the terminal;

- $E(W)$ : expected waiting time, that is, expected time that a packet waits in the queue to access the server;

- $E(R)$ : expected residual time, that is, expected remaining time to serve the packet that is in the server when a new packet arrives to the queue;

- $\rho$ : probability that the server is busy on arrival of a new packet to the queue;

- $T_{b o t}$ : back-off time (deterministic value);

- $T_{R T T}$ : round trip time;

- $T_{E S S A}$ : ESSA demodulation time [random variable (r.v.)];

- $T_{T X}$ : transmission time of a link layer packet

- $X$ : number of times to check the persistence value (probability of transmission) until the ARQ allows that the packet is transmitted (r.v.).

- $P$ : persistence index;

- $P_{t x}(P)$ : probability of transmission (persistence value) for persistence index $P$;

- $R_{i}$ : maximum transmission rate per terminal.

The terminal transmissions applying this ARQ mechanism can be modelled as an M/G/1 system, where $E(B)$ will depend on the number of link layer fragments in which the IP packet is fragmented, the number of allowed retransmissions in the system and the dimensioning of $T_{b o t}$ and $T_{A C K}$. 
For the sake of simplicity, we will assume that the system allows one retransmission only. This assumption is realistic, based on a sensitivity analysis on the impact of retransmissions in the system load carried out in the DENISE project [13] using a system level simulator based on the packet loss ratio performance figures of ESSA presented in [4]. This analysis showed that at low to medium load levels, the system never required more than one retransmission to fulfil the message loss ratio requirements (only one retransmission request was registered under an input rate of 3000 packets per second). For higher load (input packet rate of 5000 packets per second, which is very close to the congestion region in [4]), allowing one retransmission causes the system crossing the congestion line, as can be observed in Figure 3 in terms of message loss ratio versus number of retransmissions for a medium load level.

According to the preceding text, $E(B)$ can be expressed with the equation in the succeeding text, assuming that $E(B)$ represents the expected time that the process takes to transmit successfully a packet, from the moment it is scheduled to the moment that the process goes back to the scheduler to pick the next packet:

$$
\begin{aligned}
E(B)= & E\left(T_{b o t}(X+N-1)+\max \left\{T_{b o t}, T_{R x \_A C K}\right\}\right) \cdot\left(1-P_{\text {loss }}\right)^{N+1} \\
& +E\left(T_{b o t}(X+2 N)+\max \left\{T_{b o t}, T_{R x \_A C K}\right\}\right) \cdot\left(1-\left(1-P_{\text {loss }}\right)^{N+1}\right)
\end{aligned}
$$

where the number of link layer fragments per IP packet is $M=N+1$ and $E(X)$ is the expected number of times that the terminal has to check the persistence parameter to succeed getting a transmission opportunity. The r.v. $X$ can be modelled as a geometric distribution, as the problem is identical to finding the number of tosses required until the first 'head' comes up when playing 'head and tails'. Accordingly, the expected number of iterations to get a transmission opportunity is given by Equation (3), and the second moment is given by Equation (4).

$$
\begin{gathered}
E(X)=\frac{1}{P_{t x}(P)} \\
E\left(X^{2}\right)=\frac{2-P_{t x}(P)}{\left(P_{t x}(P)\right)^{2}}
\end{gathered}
$$

By the Pollaczek-Khinchin mean value formula (recalled in Equation (5)), the expected waiting time in the queue for a packet depends on the probability $\rho$ that the server is busy on arrival of the packet (defined in Equation (6) for an M/G/1 system) and the expected residual time of the packet that is currently being served, in Equation (7).



Figure 3. Message loss ratio performance for varying number of retransmissions. 


$$
\begin{gathered}
E(W)=\frac{\rho E(R)}{1-\rho} \\
\rho=\lambda \cdot E(B) \\
E(R)=\frac{E\left(B^{2}\right)}{2 E(B)}
\end{gathered}
$$

Therefore, with this model, the QoS metric $T_{S D D}$ can be expressed by the equation in the succeeding text.

$$
T_{S D D}=E(W)+E(B)-\max \left\{T_{b o t}, T_{R x \_A C K}\right\}+T_{T X}+\frac{T_{R T T}}{2}+\hat{T}_{E S S A}
$$

where $\hat{T}_{E S S A}$ corresponds to the elapsed time between the moment that the last fragment arrives at the receiver and the moment that the last missing fragment is correctly demodulated. Because the time to demodulate a fragment in the ESSA demodulator will depend on the interference level, which is uncorrelated between fragments, we can approximate $\hat{T}_{\text {ESSA }}$ by the demodulation time of any packet $T_{\text {ESSA }}$.

Equation (8) can be used to dimension system parameters to achieve a specific QoS performance or to analyse the achievable QoS performance of the system in terms of $T_{S D D}$. It shall be noted that the subtraction of $\max \left\{T_{b o t}, T_{R x_{-} A C K}\right\}$ to the sojourn time of the packet in the system is due to the behaviour of the ARQ mechanism. From the point of view of packets waiting in the queue, the serving time is $E(B)$, because this models the expected time between two consecutive transmissions. However, according to the algorithm presented in Figure 2, a packet leaves the 'server' after a time equal to $T_{b o t} E(X)-$ $\max \left\{T_{b o t}, T_{R x_{-} A C K}\right\}$. After the packet has been transmitted, the 'server' waits $\max \left\{T_{b o t}, T_{A C K}\right\}$ until it allows access to the next packet.

For dimensioning purposes, we observe the worst case waiting time for an ACK, that is, $T_{R x_{-} A C K}=T_{A C K}$ and differentiate two major cases for Equation (2) to perform the analysis of the sojourn time: case (A) $T_{b o t} \geq T_{A C K}$ and case (B) $T_{b o t}<T_{A C K}$.

(1) Case A: $T_{b o t} \geq T_{A C K}$

In this case, Equation (2) can be simplified in Equation (9), considering that both $T_{b o t}$ and $T_{A C K}$ are deterministic values specified by signalling.

$$
E(B)=T_{b o t} E(X+N)+T_{b o t}(N+1)\left(1-\left(1-P_{\text {loss }}\right)^{N+1}\right)
$$

To start with the analysis, we apply a very simple case: $T_{b o t} \geq T_{A C K}, M=1$ (all IP packets are transmitted in a single link layer fragment) and the ARQ mechanism is configured in the Tr-mode (i.e. no retransmissions are issued). In this case, Equation (2) simplifies in Equation (10); therefore, the second moment of the serving time is given by Equation (11), and the maximum transmission rate per terminal $R_{i}$ is therefore given by the inverse of the expected serving time (Equation (12)). Combining (10), (11), (6) and (7) in (5), we obtain the expected waiting time in the queue for any packet in Equation (13).

$$
\begin{gathered}
E(B)=T_{b o t} E(X) \\
E\left(B^{2}\right)=E\left(T_{b o t}^{2} \cdot X^{2}\right)=T_{b o t}^{2} E\left(X^{2}\right) \\
R_{i}=\frac{1}{E(B)}=\frac{P_{t x}(P)}{T_{b o t}} \\
E(W)=\frac{\lambda}{2} \frac{T_{b o t}^{2} E\left(X^{2}\right)}{1-\lambda T_{b o t} E(X)}
\end{gathered}
$$

Substituting Equations (10) and (13) in (8), after a few simplifications, we obtain a second order equation as follows: 


$$
\begin{aligned}
\lambda\left[E^{2}(X)\right. & \left.-\frac{1}{2} E\left(X^{2}\right)-E(X)\right] T_{b o t}^{2} \\
& -[(1+\lambda \Delta T) E(X)-1] T_{b o t}+\Delta T=0
\end{aligned}
$$

where $\Delta T=T_{S D D}-T_{T X}-\frac{1}{2} T_{R T T}-T_{E S S A}$. This can be used to dimension $T_{b o t}$ to achieve a given $T_{S D D}$ requirement (by fixing the persistence index) or to dimension the persistence index (by fixing $T_{b o t}$ ). It shall be noted that the term $\max \left\{T_{b o t}, T_{A C K}\right\}$ can be replaced by $T_{b o t}$ in case A.

It can be proven that the cases in which fragmentation and / or retransmissions are required can be expressed using Equation (14) by properly replacing the r.v. $X$ as specified in Table I.

(2) Case B: $T_{\text {bot }}<T_{A C K}$

In this case, Equation (2) can be expressed as in Equation (15).

$$
\begin{aligned}
E(B)= & T_{b o t} E\left(X+N-1+\frac{T_{A C K}}{T_{b o t}}\right)\left(1-P_{\text {loss }}\right)^{N+1} \\
& +T_{b o t}(N+1)\left(1-\left(1-P_{\text {loss }}\right)^{N+1}\right)
\end{aligned}
$$

Following the same procedure as for Case A, but applying Equation (15) instead of (9) and replacing the term $\max \left\{T_{b o t}, T_{A C K}\right\}$ by $T_{A C K}$ in Equation (8), the same analysis can be done to find the analogue expression to Equation (14) for case B. After this analysis, we obtain the following expression.

$$
\begin{gathered}
\lambda\left[\frac{1}{2} E\left((X-1)^{2}\right)-E^{2}(X-1)\right] T_{b o t}^{2}+[(1+\lambda \Delta T) E(X-1)] T_{b o t} \\
+\frac{\lambda}{2} T_{A C K}^{2}+\Delta T\left(\lambda T_{A C K}-1\right)=0
\end{gathered}
$$

It can be proven that the cases in which fragmentation and/or retransmissions are required can be expressed using Equation (16) by properly replacing the r.v. $X$ as specified in Table I. Furthermore, it can be proved that for the case $T_{b o t}=T_{A C K}$, Equations (9) and (15) are identical, as well as Equations (14) and (16). Furthermore, this model has been validated with a packet level system simulator in the DENISE project.

\subsection{Support of differentiated class of service}

The analysis performed earlier has been done assuming a first-come-first-serve queue and no service differentiation. However, the ARQ mechanism described in Section 2 can support service differentiation by setting different ARQ parameters to different service classes (also the signalling in the forward link supports differentiated parameters per service class). Obviously, the sojourn time of a packet in the system is affected if class of service is supported, as the waiting time in the queue will depend on the priority of the packet $r \geq 1$, where 1 denotes the highest priority.

The waiting time in the queue for packets of priority $r=1$ can be expressed with Equation (17). For a general case, we differentiate the input rate $\lambda_{i}$ and ARQ parameters for each service class $\rho_{i}, T_{b o t, i}, E$ $\left(X_{i}\right)$ and so on. Applying expression (17) in Equation (8), we obtain Equation (18), for $T_{b o t, 1}$.

Table I. Completion of Eqns (14) and (16) for fragmentation and/or retransmissions.

\begin{tabular}{lll}
\hline Mode & $M$ & \multicolumn{1}{c}{ Replace $X$ by } \\
\hline Tr & $N+1$ & $X+N$ \\
ACK or uACK & 1 & $X+P_{\text {loss }}$ \\
ACK or uACK & $N+1$ & $X+N+(N+1)\left(1-\left(1-P_{\text {loss }}\right)^{N+1}\right)$ \\
\hline
\end{tabular}




$$
\begin{gathered}
E(W)=\frac{\sum_{j=1}^{r} \rho_{j} E\left(R_{j}\right)}{1-\rho_{1}} \\
\lambda_{1}\left[E^{2}\left(X_{1}\right)-\frac{1}{2} E\left(X_{1}^{2}\right)-E\left(X_{1}\right)\right] T_{b o t, 1}^{2} \\
-\left[\left(1+\lambda_{1} \Delta T\right) E\left(X_{1}\right)-1\right] T_{b o t, 1}+\Delta T-\Delta r
\end{gathered}
$$

where $\Delta r$ represents the impact of the queued packets corresponding to lower priority classes, as shown in Equation (19); the queuing theory provides compact expressions for the sojourn time $E\left(S_{i}\right)$ of packets of any priority $r>1$, as in Equation (20), but deriving a similar Equation to (14) for this case becomes highly complex because of the interdependencies between the parameters of the different service classes.

$$
\begin{gathered}
\Delta r=\frac{1}{2} \sum_{i=2}^{r} \lambda_{i} T_{b o t, i}^{2} E\left(X_{i}^{2}\right) \\
E\left(S_{i}\right)=\frac{\sum_{j=1}^{r} \rho_{j} E\left(R_{j}\right)}{\left(1-\sum_{j=1}^{i} \rho_{j}\right)\left(1-\sum_{j=1}^{i-1} \rho_{j}\right)}+\frac{E\left(B_{i}\right)}{1-\sum_{j=1}^{i-1} \rho_{j}}
\end{gathered}
$$

\subsection{Dimensioning examples}

Using the equations derived from the model described earlier, the potential performance of the system in terms of $T_{S D D}$ can be evaluated for different system load scenarios and selected persistence indexes so that suitable system parameters can be selected to achieve a target $T_{S D D}$ performance. Figures 4-7 show the required dimensioning of the $T_{b o t}$ parameter (in seconds) in case A ( $\left.T_{b o t} \geq T_{A C K}\right)$ in ACK mode for different values of the persistence probability $P_{t x}(P)$ to achieve $T_{S D D}$ performance values comprised between 1 and $10 \mathrm{~s}$. Each figure shows curves for different load and fragmentation scenarios. The load scenarios range from 2.000 to 5.000 packets/s. The fragmentation scenarios are represented by the parameter $N$ as for Table I. It shall be noted that the system values in Table II have been assumed for the generation of the figures.

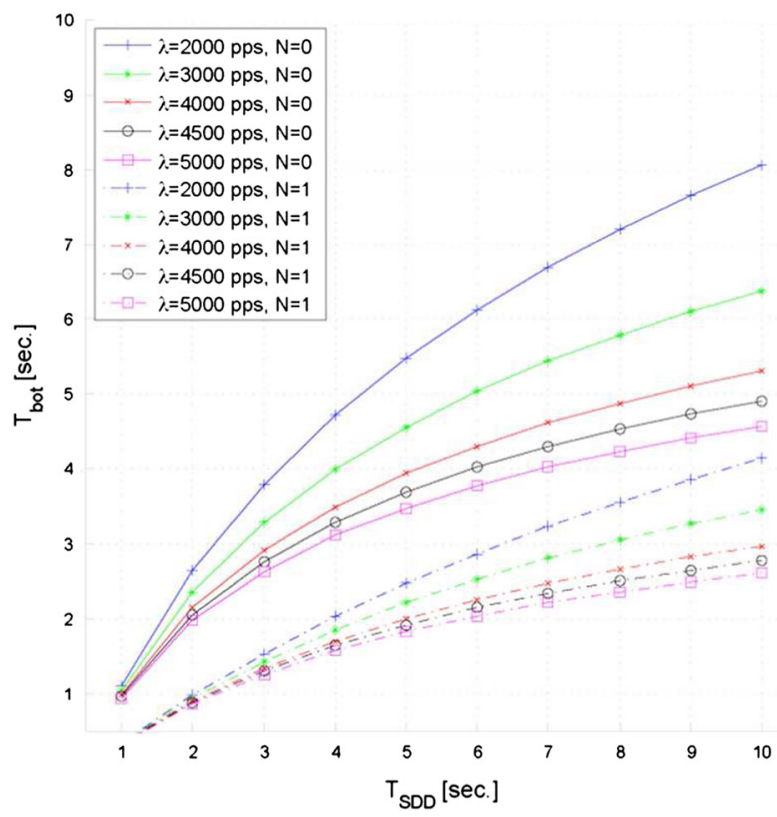

Figure 4. $T_{b o t}$ versus $T_{S D D}$ for $P_{t x}(P)=0.707$ in ACK mode. 




Figure 5. $T_{b o t}$ versus $T_{S D D}$ for $P_{t x}(P)=0.5$ in ACK mode.

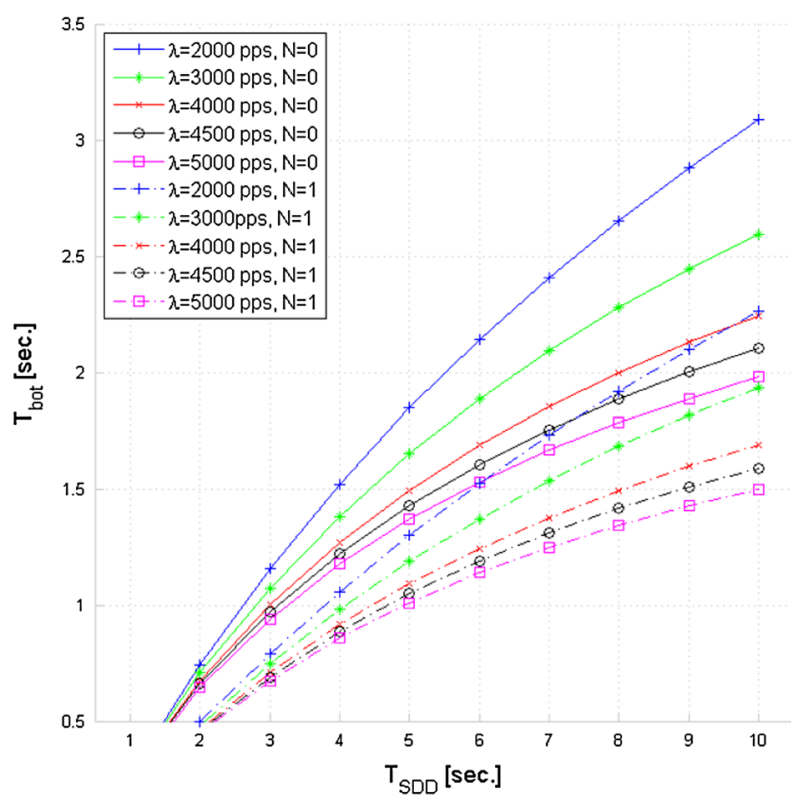

Figure 6. $T_{b o t}$ versus $T_{S D D}$ for $P_{t x}(P)=0.3536$ in ACK mode.

The work for the design and standardisation of S-MIM was jointly co-funded by the European Space Agency and Solaris Mobile Ltd.

\section{IMPACT OF ENHANCED SPREAD SPECTRUM ALOHA DEMODULATOR IMPLEMENTATION}

A first end-to-end implementation (from physical to application layer) of the S-MIM standard was realised within the SafeTRIP project [11, 12]. The objective of SafeTRIP was to create a transparent open platform to improve road safety infrastructure services. The S-MIM standard is applied in SafeTRIP to provide messaging services (in forward and return links) using low-power terminals. 


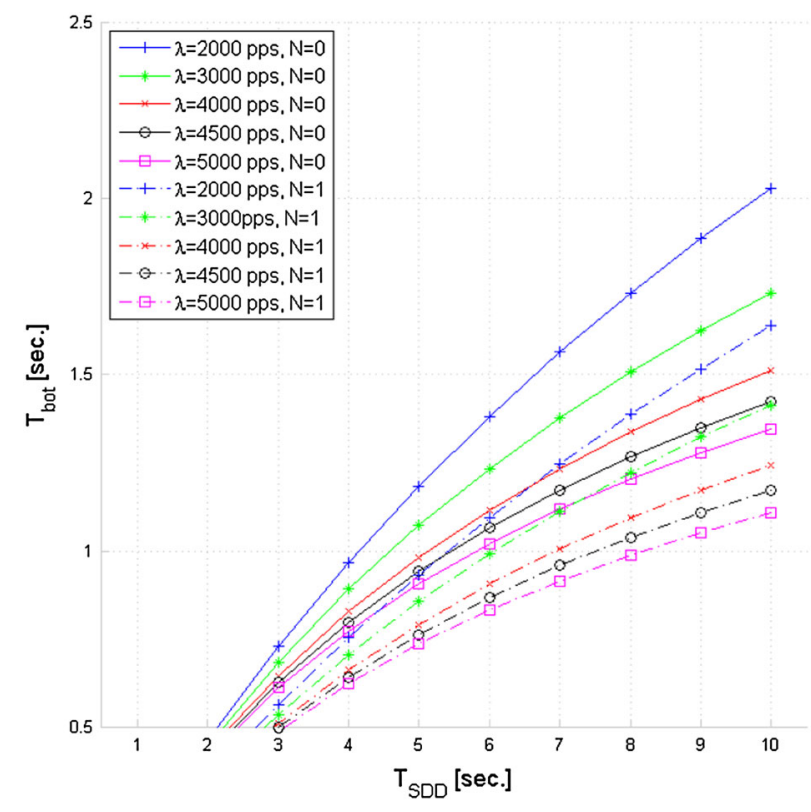

Figure 7. $T_{b o t}$ versus $T_{S D D}$ for $P_{t x}(P)=0.25$ in ACK mode.

Table II. System configuration.

\begin{tabular}{lcl}
\hline Parameter & Value & \multicolumn{1}{c}{ Comment } \\
\hline$\frac{T_{R T T}}{2}$ & $0.3 \mathrm{~s}$ & Constant \\
$T_{E S S A}$ & $N(0.3,0.06) \mathrm{s}$ & $\begin{array}{l}\text { No reliable model is available to describe the duration of the } \\
\text { IC at the satellite hub [6], as it depends on the implementation. } \\
\end{array}$ \\
& $\begin{array}{l}\text { A normal random variable has been assumed here with indicative } \\
\text { values to illustrate how to use the model. }\end{array}$ \\
& $\begin{array}{l}\text { Duration of one available ESSA frame. } \\
T_{T X}\end{array}$ & Dimensioned to keep $T_{S D D}=3 \mathrm{~s}$ \\
$T_{\text {bot }}$ & $4.75 \mathrm{~s}$ & Function of the system load according to Figure 3 in [4]. For EIRP $=2 \mathrm{dBW}$. \\
$P_{\text {loss }}$ & $f(\lambda)$ &
\end{tabular}

IC, interference cancellation; ESSA, Enhanced Spread Spectrum Aloha.

Effective Incident Radiated Power.

The link layer implementation of S-MIM in SafeTRIP is composed of two software modules, including the combined ARQ and rate control functionalities as shown in Figure 1:

- The HUB side, where the link layer reorders and decapsulates the ESSA frames detected by the ESSA demodulator into link layer packets to re-assemble IP packets. The software is constantly monitoring the incoming ESSA frames rate to estimate the current load level. This information is applied to update and transmit the S-MIM system signalling (SSA access table) in the forward link.

- The terminal side, where the ARQ and rate control algorithm depicted in Figure 2 is implemented. The transmission information and load level information are retrieved through the forward link signalling tables.

The terminal is equipped with an external power amplifier that is turned on only during the transmission phase in order to avoid interference on the forward link reception. This is a limitation of this specific hardware setup, which causes that the terminal needs a setup time of $0.2 \mathrm{~s}$ before transmitting and a holding time of $0.1 \mathrm{~s}$ after transmission. This implies of course additional delay when transmitting in the return link. This limitation could be however overcome in a more optimised commercial setup.

It shall be noted that the SafeTRIP project was developed as a proof-of-concept implementation to demonstrate the feasibility of mobile satellite services with low-power terminals. The performed tests 
were therefore neither focused on the system optimisation nor on the combined ARQ and rate control mechanism performance. However, some results gathered during the SafeTRIP project campaign drove to conclusions that are highly relevant to the focus of this paper and are therefore presented in the following.

A first set of tests were developed with the aim at isolating the terminal rate control, that is, testing the correct operation of the terminal in Tr-mode and evaluating the impact of the ESSA demodulation time in the $T_{S D D}$ performance. For this purpose, the parameter setting shown in Table II was applied, adding the $0.2 \mathrm{~s}$ delay caused by the set up time of the power amplifier to the $T_{T X}$ of $250 \mathrm{~ms}$ (which corresponds to one of the available configurations of the ESSA bursts [1]). $T_{b o t}$ was dimensioned using Equation (14) to achieve a target $T_{S D D}=3 s$ for the Tr-mode with $N=0$ and $P=1$. The terminal load was kept significantly low to assume $E(W) \rightarrow 0$ in Equation (8), that is, every packet finds an empty queue and is directly processed, whereas background load (varying interference) is applied at the demodulator side. A number of tests were carried out, varying the persistence index $P$ and the fragmentation level of IP packets. For each case, the theoretical (isolating $T_{E S S A}$ ) versus the actually achieved $T_{S D D}$ in the test environment is shown in Table III.

Figures 8(a) and 8(b) and 9(a)-(c) show the histograms of the $T_{S D D}$ performance in the laboratory tests for different background load values and two different implementations of the ESSA demodulator. It can be observed that the $T_{S D D}$ performance in the laboratory tests increases with the background interference (caused by the background traffic load), as the IC process needs to remove higher interference power to decode the packets. In fact, the ESSA demodulator implementation in

Table III. Test results.

\begin{tabular}{|c|c|c|c|c|c|}
\hline \multicolumn{3}{|c|}{ Test configuration } & \multicolumn{2}{|c|}{$T_{S D D}(\mathrm{~s})$} & \multirow[b]{2}{*}{ Maximum } \\
\hline$P$ & $N$ & Theoretical & Minimum & Mean & \\
\hline 0 & 0 & $0.6+T_{E S S A}$ & 0.6503 & 0.9451 & 1.2491 \\
\hline 1 & 0 & $1.18+T_{E S S A}$ & 1.2582 & 1.6015 & 2.0036 \\
\hline 1 & 1 & $3.03+T_{E S S A}$ & 3.1036 & 3.4260 & 3.7599 \\
\hline
\end{tabular}



Figure 8. $T_{S D D}$ histogram with the first Enhanced Spread Spectrum Aloha demodulator. 
SafeTRIP is based on software defined radio [14] with a number of IC nodes connected in cascade. Even if the thorough analysis of the ESSA demodulator performance is out of scope of this paper, it is worth noting that the cascade of IC nodes introduces a masking effect in the $T_{S D D}$ performance when the interference level is sufficiently high to require the operation of more than one IC node, as can be observed in Figure 8(b). Furthermore, a second release of the ESSA demodulator software, optimising the processing in the single IC nodes, was also tested in the laboratory showing an improved $T_{S D D}$ performance, as a higher percentage of the packets could be decoded within the first IC node for the higher background traffic case (Figure 9(b) and 9(c).

As expected, the $T_{E S S A}$ latency increases significantly with the level of interference. Furthermore, we observe two relevant effects for the dimensioning of the ARQ parameters: on the one hand, $T_{E S S A}$ highly depends on the implementation of the ESSA demodulator; on the other hand, with current stateof-the-art implementation, the order of magnitude that can be expected for $T_{E S S A}$ is significantly large with regard to other parameters that contribute to the $T_{S D D}$ performance, becoming the dominant factor with the state-of-the-art implementation. The order of magnitude of $T_{E S S A}$ will therefore play an important role in the dimensioning of the ARQ parameters.

The impact of using experimental $(E)$ values of $T_{E S S A}$ in the $T_{b o t}$ versus $T_{S D D}$ curves compared with the use of theoretical $(T)$ values of $T_{E S S A}$ can be observed in Figure 10, taking the case $P_{t x}(P)=0.707$ as indicative example for two scenarios: no fragmentation of IP packets $(N=O)$ and fragmentation of IP

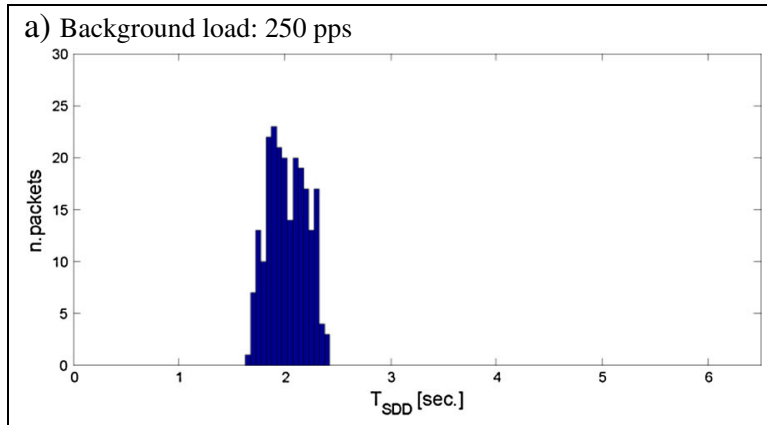

b) Backgroundload: $1000 \mathrm{pps}$

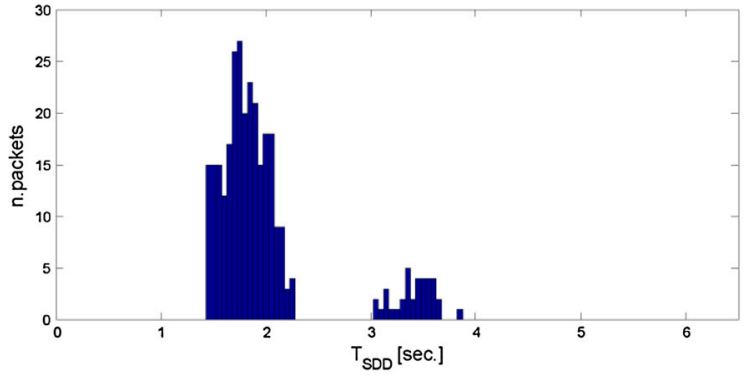

c) Background load: 3000 pps

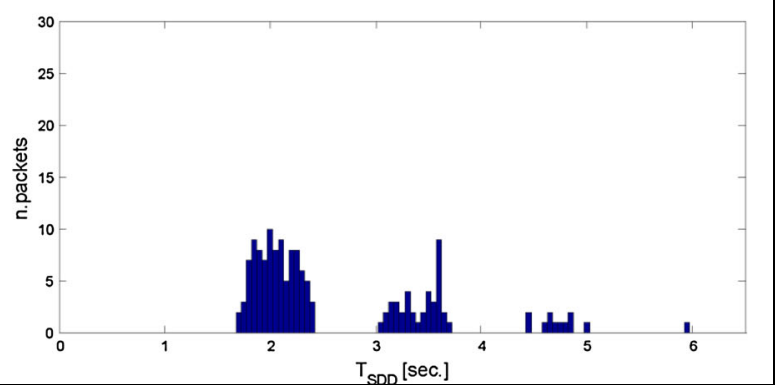

Figure 9. $T_{S D D}$ histogram with the enhanced Enhanced Spread Spectrum Aloha demodulator. 


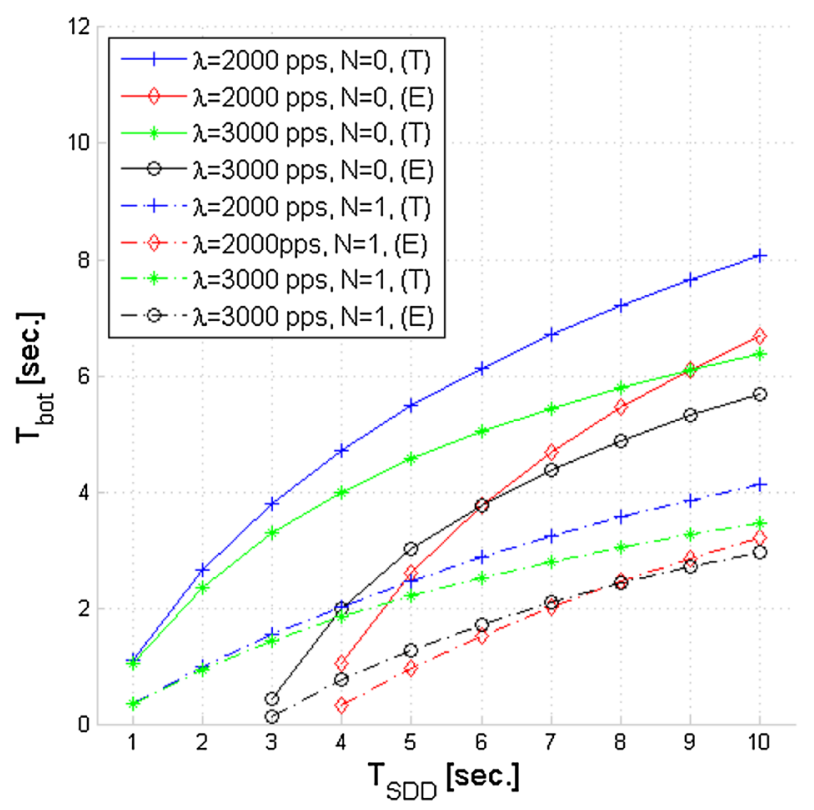

Figure 10. $T_{b o t}$ versus $T_{S D D}$ for $P_{t x}(P)=0.707$ in ACK mode with theoretical and experimental values of $T_{E S S A}$.

packets into two-link layer packets $(N=1)$. A shift of the curves is observed, limiting the achievable QoS in terms of $T_{S D D}$ and requiring a significant reduction of $T_{b o t}$ dimensioning.

\section{CONCLUSION}

The paper presents the joint ARQ and terminal rate control mechanisms defined in the S-MIM specification. A model based on queuing theory that has been presented can be applied to understand the QoS boundaries of the S-MIM system in terms of the 'Service Delivery Delay' $\left(T_{S D D}\right)$ metric and to dimension the ARQ parameters in order to achieve specific QoS guarantees.

Relevant results in terms of $T_{S D D}$ obtained in laboratory tests with the first S-MIM implementation have been presented. These results show that, with current state-of-the-art implementations of the demodulator, the demodulation time at the ESSA demodulator becomes dramatically significant already for moderate interference levels in comparison with other system parameters in the computation of the $T_{S D D}$. This effect will significantly impact the QoS boundaries of the S-MIM messaging services in the return link and must be carefully considered in the dimensioning of the ARQ parameters.

\section{ACKNOWLEDGMENTS}

The authors would like to acknowledge all the participants to the DENISE project [13], jointly co-funded by the European Space Agency and Solaris Mobile Ltd, where most of S-MIM design and related standardisation effort was carried out, and the SafeTRIP project [11], co-funded by the European Commission, where the first proof-of-concept implementation of S-MIM was achieved.

\section{REFERENCES}

1. ETSI TS 102 721. Satellite earth stations and systems (SES), Air Interface for S-band Mobile Interactive Multimedia (S-MIM). V1.2.1. Part 1 to Part 6. (2013-08).

2. Scalise S, Párraga Niebla C, Gallinaro G, et al. System design for Pan-European MSS services in S-band. 5th Advanced Satellite Multimedia Systems Conference, September 2010.

3. Scalise S, Párraga Niebla C, De Gaudenzi R, Del Rio Herrero O, Arcidiacono A, Finocchiaro D, Schlüter G, Krause J. S-MIM: a novel radio interface for efficient messaging services over satellite. in Proc. of IEEE International Conference on Communications (ICC) June 10-15 2012 in Ottawa, Canada, pp. 6953-6958.

4. ETSI EN 302 583. Digital video broadcasting (DVB), Framing Structure, channel coding and modulation for Satellite Services to Handheld devices (SH) below 3 GHz. V1.2.1. (2011-12). 
5. Vargas A, Breiling M, Gerstacker W, Stadali H, Eberlein E, Heuberger A. Adding different levels of QoS to the DVB-SH standard. in Proc. of IEEE $5^{\text {th }}$ Advanced Satellite Multimedia Systems Conference (ASMS) and $11^{\text {th }}$ Signal Processing for Space Communications Workshop (SPSC) 2010 in Sardinia, Italy, pp. 469-475.

6. Del Río Herrero O, De Gaudenzi R. A high efficiency multiple access scheme for machine-to-machine communications. IEEE Trans Aerospace Electron Systems 2012; 48(4):2961-2989.

7. Del Río Herrero O, De Gaudenzi R. A high efficiency scheme for quasi-real-time satellite mobile messaging systems. 10th International Workshop Signal Processing for Space Communications Conference, Rhodes Island, Greece, October 2008, pp. $1-9$.

8. De Gaudenzi R, Elia C, Viola R. Bandlimited quasi-synchronous CDMA: a novel satellite access technique for mobile and personal communications systems. IEEE J Selected Areas in Commun 1992; 10(2):328-343.

9. Lin S, Costello D, Miller M. Automatic repeat request error control schemes: a survey of various ARQ and hybrid ARQ schemes and error detection using linear block codes. IEEE CommMag 1984; 22(12):5-17.

10. Wissem El Bahri W, Boujemaa H, Siala M. Performance comparison of type I, II and III hybrid ARQ schemes over AWGN channels. In Proc of IEEE Intern Conf Industrial Technol (ICIT) 2004; 3:1417-1421.

11. SafeTRIP project webpage: http://www.safetrip.eu.

12. Fremont G, Grazzini S, Sasse A, Beeharee A. The SafeTRIP project: improving road safety for passenger vehicles using 2-way satellite communications. ITS World Congress 2010.

13. DENISE project webpage: http://telecom.esa.int/DENISE.

14. Heyn T, Nagel P, Niemann B, Recchia A, Collard F, Hermenier R, Marchitti MA, Andrenacci M, Mendola G. E-SSA satellite messaging return channel: performance analysis and field trials results. in Proc. of the $6^{\text {th }}$ Advanced Satellite Multimedia Systems Conference and $12^{\text {th }}$ Signal Processing for Space Communications Workshop (SPSC) in Baiona, Spain, 5-7 September, 2012.

\section{AUTHOR'S BIOGRAPHIES}



Cristina Párraga Niebla graduated in Telecommunications Engineering from Universidad Politècnica de Catalunya (UPC), Barcelona, Spain, in July 2002. She joined the German Aerospace Centre (DLR) as research scientist in 2002. Her research activities dealt with satellite-based communications system design and architectures for different application areas, such as Air Traffic Management or Public Protection and Disaster Relief, participating in several ESA and EU-funded projects. Since 2008, her main activities deal with project management in the area of civil protection. She acted recently as technical manager of the ESA DENISE project and as project coordinator of the EU-FP7 Alert4All and PHAROS projects.

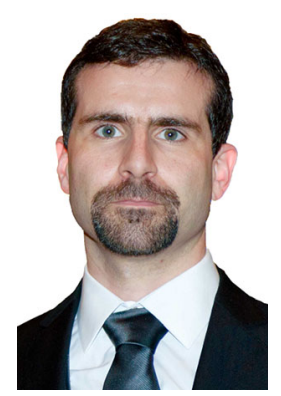

Alessandro Del Bianco studied at the University of Bologna, where he graduated the bachelor's degree in 'Telecommunications Engineering' in 2005 and master's degree in 'Electronic and Telecommunications Engineering' in 2007. Since 2007, he is working as a scientific researcher in the Institute of Communications and Navigation at the German Aerospace Center (DLR). He is mainly involved in satellite-mobile communication systems. 